\title{
Revisión general de la producción elevada de agua en la industria del petróleo
}

\author{
Angie Tatiana Ortega Ramirez; Diego Fernando Marín Maldonado; Edgar Danilo Ochoa Rodríguez \\ Grupo de Investigación Medio Ambiente y Hábitat, Manejo de aguas en la industria del petróleo, Facultad de Ingenierías, \\ Universidad de América, Bogotá, Colombia. \\ Email: *angie.ortega@profesores.uamerica.edu.co
}

\begin{abstract}
Resumen
En el presente artículo se realiza una revisión detallada sobre las características y generalidades de la producción elevada de agua en las operaciones de la industria petrolera, relacionando volúmenes de producción con los problemas técnicos que pueden estar generando dichos excesos de fluido, métodos de identificación y diagnóstico de un alto corte de agua, metodologías utilizadas por la industria tanto mecánicas y químicas para mitigar los volúmenes excesivos del recurso hídrico; para finalmente abordar el tema de la disposición final y uso de las aguas de producción de la industria del petróleo, teniendo en cuenta cifras y porcentajes.
\end{abstract}

Palabras clave: acuíferos, agua asociada, control de producción, corte de agua elevado, intrusión de agua, límite económico, polímeros.

\section{General Review of High-Water Production in Petroleum Industry}

\begin{abstract}
This article makes a detailed review of the characteristics and generalities of the high water production in the oil industry operation relating production volumes to the technical problems that may be generating such fluid excesses, methods of identification and diagnosis of a high water cut, methodologies used by the mechanical and chemical industry to mitigate the excessive volumes of the water resource. To finally address the issue of the final disposition and use of the oil industry's production waters, taking into account figures and percentages.
\end{abstract}

Key words: water intrusion, high water cutting, economic limit, aquifers, production control, polymers, water.

\section{Introducción}

En la dinámica de las operaciones de explotación y desarrollo de la industria del petróleo, la producción de agua es un tema importante, tanto como lo es la producción del crudo, debido a que los volúmenes obtenidos de agua en cada pozo, pueden determinar la viabilidad técnica, económica e incluso ambiental de su puesta en producción. Los volúmenes de agua asociada producida en superficie en la extracción del recurso fósil en los Estados Unidos son de aproximadamente 10 barriles por cada barril de petróleo que se obtiene de yacimiento (Khatib, Z. 2002), evidencia de las considerables proporciones entre los volúmenes de agua y crudo en la industria.
La situación anterior lleva a que los especialistas e ingenieros encargados de la operación petrolera deban identificar metodologías para la tipificación, mitigación, control y en lo posible eliminación de las problemáticas que llevan a la intrusión de agua al pozo productor, basados en información como historiales de producción, equipos en superficie, registros eléctricos, pruebas de pozo, registros sísmicos, entre otros, que el personal a cargo crea conveniente (Arnold, Burnett, Elphick, Feeley, Galbrun \& Otros, 2004).

Debido a la importancia del control de producción de agua en fondo, la industria del petróleo, ha desarrollado varias metodologías de control, dentro de las que destacan las de tipo mecánico y químico.

Cita: Ortega Ramírez, A. T., Marín Maldonado, D. F. y Ochoa Rodríguez, E. D. (2019). Revisión general de la producción elevada de agua en la industria del petróleo. Revista Fuentes: El reventón energético, 17(2), 39-50. 
En relación a los sistemas químicos que se pueden encontrar, algunos pueden ser la cementación forzada tradicional o cementación forzada con polímero, tratamiento con polímero para water shut off y el modificador de permeabilidad relativa; en sistemas de tratamiento basados en cementación algunos ejemplos son los Squeeze convencionales, cementos ultra fino y la cementación con espuma (Halliburton, 1996), la aplicación del tratamiento de control dependerá de las condiciones del yacimiento, pozo y operativas en las cuales se da la explotación del recurso fósil.

Aunque las metodologías descritas anteriormente permiten una mitigación en los volúmenes de agua provenientes de fondo de yacimiento, existe cierto porcentaje que llega a superficie para disposiciones adicionales. En Colombia, se busca la reutilización del agua de producción por la exploración de hidrocarburos, empleándose un volumen de 54.4 millones de metros cúbicos que equivalen a un $16 \%$ de las aguas producidas en actividades propias de la industria como el recobro secundario por medio de la inyección de volúmenes de agua, para mejorar el barrido de hidrocarburos, preparación de lodos de perforación, mantenimiento de pozos, entre otras actividades (Suárez, González, Londoño \& Pacavita, 2017), el fin principal de la reutilización del recurso hídrico es la optimización de los procesos de la industria del petróleo, la disminución de la cantidad de vertimientos y con ello la ecoeficiencia petrolera; logrando de forma simultanea aplicar una administración integral de yacimientos la cual consiste en aplicar tecnología de punta a un yacimiento conocido dentro de un entorno de gestión dado con el fin de maximizar las ganancias del yacimiento optimizando la producción del hidrocarburo y minimizando tanto la inversiones de capital como los gastos operativos (Martinez \& Konopczynski, 2002).

\section{Metodología}

Para el desarrollo del presente artículo se realiza una búsqueda detallada de información mediante Google Scholar, Researchgate, tesis académicas, congresos técnicos, normas técnicas y políticas, entre otros; con una posterior clasificación de enfoques, información y citas, que respalde la investigación realizada por los autores en relación a la producción elevada de agua en la industria del petróleo.

\section{Problema inicial}

La producción de hidrocarburos dentro de un pozo de petróleo, se encuentra acompañado de la extracción de volúmenes de fluidos adicionales como agua y gas, considerándose un sistema de flujo trifásico, donde fluye gas, petróleo y agua (Pérez, Santos Santos, \& Rojas, 2010). Éstos fluidos generalmente se producen mediante cinco mecanismos de producción primaria: gas en solución, capa de gas, segregación gravitacional, expansión de roca y fluidos; y empuje hidráulico (Paris de Ferrer, 2001). El empuje hidráulico es el mecanismo de producción primaria predominante y es aquel que permite una producción más duradera, debido a que el agua se encuentra en la mayoría de campos petroleros, es el fluido más abundante en el yacimiento y con ello se evita el depletamiento acelerado del mismo (Kunchuk, Sengul, \& Zeybek, 1999), es decir, el mecanismo de producción primaria por empuje hidráulico permitirá la extracción de fluidos del yacimiento generando una caída de presión lenta y por consiguiente una producción de hidrocarburos con una declinación lenta y constante a lo largo de la producción primaria del pozo.

El agua de producción hace referencia a la reportada en superficie proveniente de la producción de pozos de hidrocarburos, desde la zona de interés (agua connata), un acuífero activo adyacente (agua de intrusión) o un proyecto de inyección de agua (agua inyectada), (Ebratth Salgado, 2011) (Cruz, Duque \& Marulanda, 2015).

El agua de inyección proviene de un pozo tipo inyector o de un acuífero que se encuentra activo y ayuda al barrido del petróleo líquido en un yacimiento (Kunchuk, Sengul, \& Zeybek, 1999).

El agua es un fluido que se encuentra presente durante toda la vida productiva de un pozo y por ello es importante identificar si el volumen de agua producida afecta la viabilidad financiera del proyecto de extracción del hidrocarburo, por tal razón se encuentran dos tipos de agua de producción en un pozo de petróleo los cuales se mencionan a continuación:

- El agua buena es aquella que se produce por debajo del límite económico de la "Water-Oil Ratio" (Rodríguez González, Cabrera Pilco, \& Condo Ramos, 2014). No se puede controlar sin perder también flujo de hidrocarburos (Baily, y otros, 2000).

- El agua mala, es aquella que se produce sobre el límite económico dado por el Water-Oil Ratio (WOR), por lo cual, la producción de petróleo es tan baja con respecto a la producción de agua que no es económicamente rentable para compensar costos de tratamiento en superficie (Baily, y otros, 2000). 
Los altos volúmenes de producción de agua es un tema relevante para la industria del petróleo, por ejemplo para el año 2004, Schlumberger reporta una producción promedio de 3 barriles de agua por cada barril de petróleo a nivel mundial, lo cual implica una inversión de cuarenta mil millones de dólares al año (Baily, y otros, 2000); mientras que, para el año 2016, Ecopetrol muestra una producción promedio de 11,6 Barriles de agua por cada barril de petróleo en Colombia (Suarez, Jaramillo, Gonzaléz, Londoño, \& Pacavita, 2016). Es decir que para el sector del petróleo en Colombia la producción de agua es bastante elevada con respecto a la cantidad de petróleo extraído, situación que afecta de manera directa y negativamente la rentabilidad de los proyectos de inversión para la industria, por los altos costos de tratamiento.

El límite económico de un proyecto de explotación en un yacimiento, se define como el momento en que ya no se hace rentable económicamente la extracción de los fluidos que se encuentran almacenados en éste, debido a factores como la excesiva producción de agua o producción de hidrocarburos a muy bajos caudales; lo cual, no genera las utilidades económicas suficientes para sustentar el mantenimiento del proyecto (Ahmed, 2010). El límite económico generalmente se define mediante la Relación AguaPetróleo (WOR, en inglés); una gráfica de la relación agua-petróleo vs. Tiempo, permite evaluar cuando ocurre un problema de excesiva producción de agua (Chan, K.S., 1995). Se dice que en los diagramas donde se grafica WOR y su derivada a lo largo del tiempo, se puede distinguir si un pozo experimenta un avance prematuro del agua causado por conificación de agua o la canalización a través de capas de alta permeabilidad (Chan, K.S., 1995). Sin embargo, se recomienda el uso de métodos adicionales de diagnóstico para los mecanismos asociados a la producción excesiva de agua, para cerciorarse de su afectación al pozo evaluado (Seldal, M., 1997).

El artículo comprende una revisión bibliográfica, que permite revisar el tema sobre la producción excesiva de agua (agua mala), identificando el mecanismo de intrusión al pozo productor y una descripción de tratamientos empleados actualmente, analizando sus impactos técnicos y económicos en relación a rentabilidad del proyecto.

A continuación, se enumerarán los principales mecanismos de intrusión de agua en orden de complejidad al solucionarlos:
- Filtraciones en el revestimiento, tuberías de producción o packers: se genera una filtración de agua proveniente de zonas que no son productoras de hidrocarburos mediante canales presentes en el revestimiento, tuberías de producción o packers.

- Flujo canalizado detrás del revestimiento: Fallas en la cementación primaria que conectan zonas acuíferas con zonas de producción de hidrocarburos, dicha fallas se generan principalmente por una descentralización de la herramienta de cementación o por un mal desplazamiento del lodo al realizar dicha operación.

- CAP dinámico: La intrusión de agua se genera debido a una muy baja permeabilidad vertical que origina un ascenso del contacto agua petróleo hacia la zona abierta del pozo.

- Capa inundada sin flujo transversal: Se produce por zonas de alta permeabilidad que se inundan con agua de acuíferos adyacentes o por la presencia del agua de inyección.

- Fracturas o fallas entre inyector y productor: un pozo usado para inyección con fracturas en el fondo, puede tender a producir rápidamente la misma agua en el pozo productor.

- Fracturas o fallas de una capa de agua: el agua puede venir de fracturas que se encuentran conectadas a un acuífero subyacente, interceptando las fracturas mediante las cuales se está haciendo la extracción del Hidrocarburo.

- Conificación o formación de cúspide (Cusping): generalmente se produce en pozos verticales cuando el CAP está cerca de los disparos en una formación con permeabilidad vertical alta.

- Barrido areal deficiente: es un mecanismo de intrusión de agua causado por la anisotropía de las permeabilidades del yacimiento.

- Segregación gravitacional: se genera al encontrarse una capa ancha con buena permeabilidad vertical y se produce la invasión de agua no deseada. El agua va hacia el fondo por efecto de gravedad, pero va subiendo el nivel del CAP debido a la permeabilidad vertical que se considera buena.

- Capa inundada con flujo transversal: este mecanismo de intrusión del agua es similar a la capa inundada sin flujo transversal. Pero se diferencia en 
que éste no tiene una barrera que detenga el flujo en el yacimiento. Es más difícil de controlar este efecto comparado con la capa inundada sin flujo transversal.

\section{Métodos de identificación y diagnóstico de un alto corte de agua:}

La industria del petróleo emplea registros de producción que permiten evaluar el movimiento del fluido dentro y fuera del pozo, especificando mediante la interpretación y correlación los intervalos de entrada de agua (Mukerji, 2013). Los registros de producción son una herramienta esencial para el manejo del yacimiento a lo largo de su vida productiva, puesto que facilita la recopilación de información y con ello la identificación y solución de problemas que generan una reducción en la producción de petróleo; sin embargo, en la industria petrolera la mayoría de los registros de producción se corren para identificar el ingreso de fluidos al pozo productor permitiendo discriminar entre agua, gas y petróleo facilitando de esta forma identificar la presencia grandes volúmenes de agua no deseada, debido a que aumenta los costos de producción del hidrocarburo y genera gastos adicionales al momento de tratarse y disponerse de manera segura, por tal motivo es importante localizar la fuente de la producción de agua para así lograr una planificación optima de método de control de producción de agua (Sondex Wireline Ltd, 2006).

A menudo se realizan estudios de trazadores Interwell, los cuales proporcionan caracterizaciones muy valiosas y a su vez económicas de reservorios fracturados, especialmente para juzgar la aplicabilidad de los tratamientos con gel para disminuir la canalización (Wagner, O.R., 1977). Los datos obtenidos a partir de estos estudios proporcionan una resolución mejor de las heterogeneidades del yacimiento que los análisis de gradientes de presión (Datta-Gupta, A., Vasco, D.W., and Long, J.C.S., 1995), garantizando una caracterización más detallada y precisa del yacimiento.

Los resultados de los trazadores interwell indican si las fracturas están presentes y si son la causante de que se esté produciendo canalización de agua, la ubicación y dirección de los canales, el volumen de las fracturas, la conductividad de la fractura y la efectividad de un tratamiento reparador (Smith, L.R., Fast, C.R., and Wagner, O.R., 1969). Por lo cual, los trazadores interwell resultan ser uno de los mecanismos más completos de diagnóstico de una producción de agua, generando una serie de información de gran utilidad para la selección de posibles tratamientos en búsqueda de controlar la producción de agua directamente en la fuente generadora.

Con respecto al análisis de la producción de agua, se describen a continuación algunos de los registros de producción empleados para localizar las zonas de intrusión del agua en los pozos productores:

Registro de temperatura: es una herramienta utilizada para medir la temperatura del pozo a diferentes profundidades por medio de sensores de temperatura conectados a la herramienta, observando así los intervalos en los cuales se presenta una variación de temperaturas por el ingreso de líquidos o gas a través de fracturas, este efecto también se genera por la filtración del fluido de perforación a la formación (Glover, 2008).

Medidores de tasa de flujo (flowmeters): permite evaluar las tasas de flujo, registrar el perfil de flujo y el porcentaje de producción de cada una de las zonas activas presentes, discriminando entre zonas productoras o ladronas de fluido en el pozo productor, evaluando la integridad del pozo e identificando fracturas o fugas de fluido. Hay tres tipos de medidores: continuo, de empaque y de diámetro total (Porras Tirado \& Suarez Medina, 2016).

Registro gradiomanómetro: mide los cambios en el gradiente de presión identificando la naturaleza de los fluidos presentes en el pozo, mediante la medición de la diferencia de presión entre dos sensores espaciados a dos pies. El registro gradiomanómetro se puede escalar en unidades de densidad de fluido diferenciando entre agua, aceite o gas (Lazarde, 2004).

Registro sónico compensado (BHC): mide la propagación del sonido con múltiples transmisores y receptores que emiten una señal sónica y registran sus impulsos respectivamente. La onda emitida por la herramienta se propaga a través del lodo a la formación chocando con las paredes del pozo y dentro de la formación para llegar a los receptores de la herramienta (Moreno Vázquez, 2011).

Registro de cementación (CBL): permite realizar un perfil de Adherencia de cemento mediante la propagación de un pulso acústico emitido por un transmisor a través de los materiales que rodean al instrumento, para que los dos receptores ubicados en la herramienta midan la amplitud y el tiempo de viaje de la onda acústica. Este registro se realiza con el fin de determinar la presencia de cemento entre el casing 
y la formación y su grado de adherencia, identificando posibles zonas de canalizaciones de fluidos (Espinoza Okamoto, 2007).

Registro de densidad variable (VDL): indica la calidad de adherencia del cemento al representar la amplitud de la onda recibida mediante una matriz variable del blanco al negro en la cual el color negro representa una amplitud de onda positiva, el blanco una amplitud negativa y el gris representa la intensidad de la amplitud positiva (Espinoza Okamoto, 2007).

Al detectar una zona en la que está ingresando volúmenes de agua, es importante identificar el corte de agua que presenta el pozo productor. Actualmente se han desarrollado analizadores de corte de agua que proporcionan una medida del contenido de agua en la mezcla agua-petróleo a condiciones de flujo, sin ser necesario una separación de estos fluidos antes de su medición (ERCB, 2003). Algunas técnicas de medición del corte de agua son:

- El medidor de corte de agua Haimo FM 2000 es una tecnología de rayos gamma de energía dual para la determinación de las fracciones de fases como el contenido de agua y la fracción de volumen de gas en una mezcla de tres fases (agua, petróleo y gas) (Khamis \& Haridas, 2003).

- El medidor de corte premier (Red Eye) es un medidor de corte de agua que emplea tecnología basada en la emisión masiva de radiación infrarroja a través del fluido producido (agua-petróleo). Esta herramienta utiliza el principio básico de espectrometría en el cual se estima que la diferencia de absorción de radiación infrarroja entre el petróleo crudo y el agua (Khamis \& Haridas, 2003).

- El medidor de corte de agua en línea que utiliza tecnología de resonancia magnética nuclear en campo es una solución para medir el corte de agua en un sistema de petróleo pesado donde instrumentos convencionales presentan dificultades por la presencia de una alta gama de emulsiones y donde la salinidad del agua producida puede sufrir variaciones significativas (Lastockin, Wright, Allsopp, Dakers, \& Kantzas 2004)(Martin \& Páez, 2017).

\section{Posibles soluciones}

El control de producción de agua en un pozo se puede realizar durante la etapa de completamiento del pozo o durante su vida productiva, en la cual el agua producida supera el límite económico. Varios artículos han abordado la selección de pozos candidatos y diversos aspectos a tener en cuenta a la hora de elegir el tratamiento para un problema específico asociado a la producción de agua (Soliman, M.Y. et al, 2000), los cuales se hablarán a continuación. Existen metodologías (mecánicas y químicas) utilizados para el control de la producción de agua que se encuentran sujetos de aplicación al tipo de problema que se presenta en el pozo (Cortes, V.M., 2008):

Las estrategias mecánicas comprenden el empleo de sellos mecánicos, tapones de arena, estranguladores de fondo y tapones de cemento (squeez) (Cortes, V.M., 2008), dando solución a problemas asociados a filtrados en la tubería de revestimiento, conificaciones y flujos detrás de la tubería de revestimiento.

Los sellos mecánicos se instalan dentro del pozo con el fin de aislar las arenas de una zona del yacimiento del pozo productor y bloquear su producción por debajo de ese intervalo (Cortes, V.M., 2008). La cementación forzada es una técnica de cementación remedial en la cual se desplaza la lechada de cemento a altas presiones hacia un área específica del pozo detrás de la tubería del revestimiento (Cortes, V.M., 2008), con el fin de sellar canales generados por un mal desplazamiento del lodo o una mala centralización durante el procedimiento de cementación primaria del pozo. Los estranguladores de fondo aplican una contrapresión que aumenta de forma artificial la presión dentro del pozo (Cortes, V.M., 2008), retardando el flujo de agua y aumentando la recuperación de petróleo debido a que genera un frente más homogéneo, reduciendo la probabilidad de generarse una conificación. Finalmente, los tapones de arena consisten en ubicar arena en el fondo de pozo (Cortes, V.M., 2008), con el objetivo de reducir o cerrar en su totalidad la producción de un intervalo del pozo.

Las soluciones químicas consisten en la inyección de polímeros que alteran las propiedades de las rocas (Cortes, V.M., 2008), reduciendo la capacidad de flujo del agua o generando una barrera que evite el paso del agua sin afectar la producción de petróleo (polímeros hidrofílicos) (Molano, Navarro \& Díaz, 2014). Entre este tipo de tratamientos químicos encontramos: bloqueadores de permeabilidad selectivos o no selectivos (gelificantes), reductores desproporcionados de permeabilidad y modificadores de permeabilidad relativa. Por ejemplo, los geles rígidos, se pueden preparar a partir de varios materiales que producen 
la disminución de permeabilidades en bajos mDarcy (Krishnan, P. et al, 2000). Para este caso, es necesario preparar el gel con un rango entre el $4 \%$ y $7 \%$ de polímeros de acrilamida para obtener un peso molecular relativamente bajo $\mathrm{y}$, por lo tanto, también posea una baja viscosidad (Syndask, R.D., 1993).

En relación a los mecanismos químicos de control se puede tener los tratamientos selectivos o no selectivos.

- Los tratamientos selectivos: hacen referencia a productos químicos que interactúan selectivamente con el agua, reduciendo la permeabilidad relativa al agua (Krw) aumentando la mojabilidad de la roca hacia el agua, permitiendo que haya una mayor movilidad de los hidrocarburos a través de la roca., de este tratamiento se encuentran tres clases:

- Bloqueadores de permeabilidad selectivos. Consisten en el uso de un surfactante viscoelástico aniónico, (VAS) que, en presencia de cationes, produce geles de baja viscosidad a altas velocidades de corte (Cortes, V.M., 2008). Los hidrocarburos rompen el bloqueador al pasar por él, y se revierta la viscosidad del sistema, haciendo que la roca quede humectada por agua y los hidrocarburos puedan producirse libremente.

- Reductores desproporcionales de permeabilidad. Los materiales de éstos reductores taponan el volumen poroso, pero detienen el paso de agua y el de hidrocarburos casi en igual proporción (Cortes, V.M., 2008). Es decir, los reductores desproporcionales de permeabilidad sellan el flujo tanto de agua como de hidrocarburos. Para éste polímero se usan reactivos como: derivados de resina de madera, tetra metil ortosilicato y etil silicato, el cual reacciona en presencia de agua y forma un gel de sílice (Sun, Crouse, Freed, Xu, Bautista, Zhang \& Dressler, 2018).

- Modificadores de Permeabilidad relativa (PRM). Se trata de polímeros hidrofílicos que, al contacto con el agua, ocupan el espacio de volumen poroso (Cortes, V.M., 2008). Teniendo como efecto final la reducción de la permeabilidad relativa del agua y alterando en menor proporción la permeabilidad relativa al petróleo, mejorando la movilidad de los hidrocarburos.

- Tratamiento No-Selectivo: no se distingue el tipo de permeabilidad que se va a ver reducida. Es decir que es muy parecido a un sello mecánico, en el cual se detiene la producción de agua o hidrocarburos indiscriminadamente.
- Bloqueadores de permeabilidad o Gelificantes. Tapan el volumen poroso y evitan el flujo de fluido debido a una reacción química retardada. Se usan reactivos como el Silicato de Sodio, activadas con ésteres de urea o resinas aminoplásticas (Cortes, V.M., 2008). Generalmente son controladas por medio de pH. Forman un gel de alta obstrucción, tapando poros que contienen aceite y/o agua casi en su totalidad.

Aparte de las soluciones mencionadas anteriormente, se ha hecho varios estudios para tratar, prevenir o corregir los causales de la elevada producción de agua definidos anteriormente. A continuación, se mencionan algunas de las soluciones para pozos que presentan una excesiva producción de agua con respecto a la de petróleo, haciendo énfasis en las causas que más se encuentran en la industria.

- Para filtraciones en el revestimiento, tuberías de producción o packers, se involucran procedimientos de cementación (Meek, 1991), o parches mecánicos (Macrae, 1997). Generalmente los tapones de cemento se desalojan fácilmente con la presencia de pequeños golpes mecánicos. Los tratamientos con gel, pueden ser más exitosos a la hora de resolver este tipo de problema (Urdahl, 1992). Es recomendable el uso de un gel resistente y de permeabilidad baja, el cual permita el taponamiento de fugas (Seright, 1993) para evitar el posterior ingreso de agua por medio de microfracturas.

- Para el flujo canalizado detrás del revestidor, generalmente se realizan tratamientos con tapones de cemento (Marca, 1990). El tratamiento con cemento es excelente, siempre y cuando los canales que se conectan entre el revestidor y la formación no sean muy estrechos. Sin embargo, si se tienen canales estrechos que permitan el flujo detrás del revestidor, la opción más viable es el uso de geles, puesto que se fijan de una manera más versátil que el cemento. La capacidad de los geles para resistir gradientes de alta presión aumenta cuando disminuye el ancho del canal (Seright, R.S., 2000); por lo tanto, no se espera que el gel tape por si solo los huecos grandes detrás de la tubería (Lai, Q.J., et al, 1999), a veces resulta muy útil la inyección de polímero y posteriormente la inyección de una lechada de cemento para evitar que el gel se filtre fuera de sus ubicaciones estratégicas.

- En el caso de una capa inundada sin flujo transversal, se usan tapones de cemento o de arena para detener la producción de agua cuando 
el acuífero se encuentra en el fondo del pozo. En cambio, cuando el acuífero se encuentra arriba de una zona petrolera, se incluyen cementos, carbonatos, empaquetadores o parches mecánicos (Marca, 1990); sin embargo, la inyección de geles también se usa para el tratamiento de este problema (Fulleylove, 1996), ya que los geles generalmente se aplican para taponar zonas y evitar el flujo de fluido.

- Con respecto a las fracturas o fallas entre productor e inyector, algunos de los tratamientos más exitosos fue la aplicación de un gel usado para reducir la canalización de agua y gas en depósitos (Hild, 1999). El objetivo principal de este método es mejorar la eficiencia de barrido, y promover la producción de petróleo incremental. En las fracturas o fallas de una capa de agua, los tratamientos con Gelant causan un aumento sustancial en la producción de agua. Estos tratamientos con gel se basan en la capacidad del gel para colocarse en una matriz rocosa (Seright, 1998) y, por consiguiente, reducir la permeabilidad al agua en mayor medida que la permeabilidad a los hidrocarburos.

- En una situación de conificación se recomienda la disminución en el diferencial de presión (Drawdown) mediante la producción de fluidos a un caudal más bajo, puesto que los tratamientos con geles tienen una probabilidad muy baja de éxito cuando se trata un problema por formación de cúspide, además de ser tratamientos costosos según la composición del polímero. Se tiene la idea errónea de que el gel solo ingresará a la zona de agua en el fondo de pozo, pero en realidad esto pasaría si el crudo tiene una gravedad API muy baja (tiene una viscosidad extremadamente baja), varios estudios han demostrado que para que el tratamiento con geles o polímeros sea exitoso en un problema de conificación o formación de cúspide, se debe disminuir la tasa de producción es 1,5 a 5 veces menor a la tasa crítica de pretratamiento (Faber et al., 1998) para evitar que el gel inyectado sea barrido por un caudal de flujo alto.

- En una capa inundada con flujo transversal, se espera que a partir del uso de geles se tenga un bajo nivel de eficiencia (Sorbie et al., 1992), ya que incluso si el gel se limita solo al canal de alta permeabilidad, el agua buscará fluir rápidamente por cualquier espacio relativamente pequeño. La única forma de que sea eficiente es poner un tapón muy grande de manera selectiva, que permita sellar toda la zona de alta permeabilidad (Root, 1965) pero resulta muy costoso y también afecta el ingreso de hidrocarburos al pozo.

\section{Consecuencias}

Mundialmente, se produce en promedio tres barriles de agua por cada barril de petróleo (Bailey et al, 2000). El agua se encuentra presente en todos los campos petroleros y es el fluido más abundante en éstos (Kunchuk, Sengul, \& Zeybek), presentándose generalmente en campos maduros, es decir, campos que han sido explotados durante varios años.

A pesar del arduo trabajo y esfuerzo por parte de las empresas de servicios para el control de producción de agua en superficie y fondo de pozo, el agua de producción sigue siendo un subproducto necesario y complicado de manejar en la producción de petróleo y gas ya que en gran medida, la relación agua-petróleo es la que define el límite económico para la explotación de hidrocarburos en ese pozo (Veil, Puder, Elcock \& Redweik, 2004), ésta relación se calcula dividiendo la producción de agua entre la producción de petróleo, es decir que si se obtiene una RAP alta en un pozo, se está produciendo agua en mayor proporción y es deber del ingeniero predecir hasta qué punto es viable económicamente extraer hidrocarburos en ese pozo y a que tratamientos se puede someter el pozo.

Por lo tanto, para lograr un alto porcentaje de éxito al momento de tratar los problemas de producción de agua, primero debe identificarse correctamente la naturaleza del problema (Elphick, J. and Seright, 1997), esto mediante los registros eléctricos y métodos de diagnóstico mencionados anteriormente.

Generalmente se usan cálculos simples por medio de la ecuación de Darcy con un enfoque para la solución de problemas asociados a la producción de agua de flujo lineal, ya que también se puede dar un enfoque donde el flujo es radial; sin embargo, es más fácil realizar un tratamiento con geles en una consideración de flujo lineal debido a su menor complejidad y el uso de un volumen óptimo de gel a colocar (Seright, R.S., 1988). De esta manera, se decide inferir que el problema está asociado a la producción de agua de forma lineal para disminuir el tiempo en que el profesional decide el tratamiento por el cual someterá al pozo.

Con lo anterior se deduce que, si no se tiene un amplio conocimiento en la solución de problemas relacionados a los mecanismos de producción excesiva de agua, se va a 
generar pérdida de dinero y tiempo, puesto que se puede implementar la solución enfocada a un tipo específico de mecanismo, y éste no podría ser el principal factor por el cual se presenta el elevado corte de agua.

Además, si se sigue produciendo el agua a la medida que se produce el petróleo y no se controla en fondo, acarreará costos de levantamiento para llevarse a superficie, separación de agua libre, eliminación de trazas de crudo, filtrado, bombeo e inyección (Bailey, B., et al, 2000), además de la posterior adecuación de las propiedades del agua dependiendo de la disposición final que se le otorgue en el futuro (disposal, riego, entre otros).

\section{Disposición y usos del agua en exceso}

El agua producida simultáneamente durante la producción de hidrocarburos es sometida a diversos tratamientos los cuales buscan reducir la concentración de los contaminantes presentes en este fluido para dar cumplimiento con la normatividad que regula la disposición del agua producida. Algunos de los principales contaminantes son: el contenido de sólidos, aceites y grasas, compuestos orgánicos disueltos y volátiles, metales pesados, radionucleidos, bacterias disueltas, productos químicos (aditivos), entre otros. (Society of Petroleum Engieer, 2011), los cuales al no ser tratados correctamente generan afectaciones significativas sobre las fuentes hídricas o superficies donde son dispuesto afectando directamente los ecosistemas presentes en estos territorios.

Uno de los métodos más común de disposición consiste en descargar el agua producida en fuentes hídricas superficiales luego de ser tratada para cumplir con los parámetros establecidos por la norma, sin embargo, no es posible verter en su totalidad el agua producida, por lo cual en algunas ocasiones parte de este volumen de agua es descargado en superficie, rociando los caminos en invierno y aprovechando su salinidad para derretir el hielo, (Gomez Angeles, 2011) garantizando una reutilización de este fluido de forma sostenible y ambientalmente amigable basados en sus propiedades para lograr un beneficio a una comunidad ya sea mediante actividades complejas o simplemente humedeciendo carreteras evitando la propagación de polvo que afecte la salud de las personas.

Otra de las opciones de disposición final del agua producida consiste en la inyección en el subsuelo, ya sea para recuperación secundaria del petróleo buscando aumentar la producción del hidrocarburo en yacimientos productivos los cuales han sufrido un depletamiento generando una caída en su producción (Carrillo Pérez, 2010) o simplemente para su disposición en pozos de disposal. Sin embargo, en diversos campos productores han decidido inyectar el agua de producción en acuíferos para almacenamiento y posterior uso de este fluido o simplemente para un propósito hidrográfico en el cual buscan controlar la subsidencia o hundimientos (Galvis Briñez, 2011).

No obstante, la industria petrolera ha buscado reutilizar el agua de producción en diferentes procesos y actividades tanto a nivel agrícola como industrial, aprovechando al máximo este fluido para la recuperación de zonas mediante el riego de cultivos o simplemente ser utilizada para operaciones de perforación o fracturamiento hidráulico. Sin embargo, es importante destacar que la mayoría de oportunidades para la reutilización del agua producida se generan en las operaciones de petróleo y gas (Colorado Energy Office \& University Water Center, 2014), esto se debe a la búsqueda constante por parte de la industria petrolera de aumentar el nivel de producción de petróleo, por lo cual se decide reutilizar el agua producida para ser inyectada y de esta forma aumentar el factor de recobro.

El agua de producción ha sido utilizada a gran escala en el sector agrícola tanto para el revestimiento de zonas áridas como para el riego de cultivos hidropónicos y de pastoreo con fines de alimentación ha ganado (Galvis Briñez , 2011). El uso del agua de producción en el sector agrícola se ha convertido en una nueva tendencia de uso eficiente y responsable del agua producida, generando grandes beneficios en zonas donde se ha desarrollado proyectos agrícolas utilizando el agua de producción, continuación se mencionarán algunos de estos proyectos agrícolas:

- Reverdecimiento del desierto 103A North Camp Q5 ubicado en el desierto de Libia, a unos $3000 \mathrm{Km}$ al sureste de Benghazi mediante humedales como parte del tratamiento y sembrando Phragmites australis nativas de dicho desierto. El volumen de agua de producción tratada en esta Proyecto fue de 377 -503 bbl/día (Guirguis, 2004) (Díaz, Zamora, Caselles-Osorio \& León, 2013).

- Uso del agua producida en yacimientos petrolíferos en calidad de agua de riego/ agua potable. Este proyecto utilizo una combinación de plantas de cultivos hidropónicos y una captación aproximada de 50.000 bbl/día (F.T. Tao, y otros, 1993).

- Uso del agua asociada a la producción de Metano en la actividad de riego en la cual se 
usaron $12500 \mathrm{bbl} /$ día con el objetivo de cubrir aproximadamente 40.5 hectáreas de terreno árido (Galvis Briñez, 2011).

Actualmente se ha determinado que el agua de producción puede ser reutilizada para el desarrollo de productos químicos, tales como la soda caustica debido a que dicho fluido está compuesto principalmente por cloruro de sodio y el proceso industrial de la soda caustica se basa en las salmueras. La soda caustica es una materia prima importante para una amplia variedad de productos tales como el jabón, cosmético, entre otros, por lo cual producirla a base del agua producida con alta salinidad podría representar grandes beneficios económicos y ambientales al utilizar el $\mathrm{CO}_{2}$ capturado de las emisiones atmosféricas. Este método de reutilización ha sido experimentado a escala de laboratorio y se ha demostrado que el proceso es factible (Carvalhinho Grimaldi, y otros, 2010), demostrando de esta forma que el agua de producción presenta grandes propiedades que pueden ser aprovechadas en procesos químicos, convirtiéndose en materia prima para la obtención de diversos productos como la soda caustica.

\section{Conclusiones}

De acuerdo a la temática revisada a lo largo del artículo, se puede concluir que el volumen de agua producido por la industria del petróleo en su operación de extracción de residuo fósil es considerablemente alto y por ello requiere de metodologías o estrategias que permitan disminuir su valor de producción desde fondo de pozo que permita una mayor rentabilidad dentro de los proyectos desarrollados.

El agua producida desde el yacimiento a superficie no es totalmente "mala", debido a que este fluido es necesario como mecanismo de empuje ya sea primario o secundario (acuífero o perfiles de inyección) para llevar el crudo o superficie, esta agua es denominada como el "agua buena" y ayuda a cumplir el fin último de la industria. La producción de fluidos del yacimiento, especialmente la relación agua - petróleo es la que determinar el limite económico de un pozo y con ello si es viable su explotación o no.

Existen diversos problemas en fondo de pozo que pueden originar la intrusión elevada de agua a los canales de producción como filtraciones en el revestimiento, flujo canalizado detrás del revestimiento, CAP dinámico, capa inundada sin flujo transversal, fracturas, conificaciones, barrido areal deficiente, segregación gravitacional, capa inundada con flujo transversal, siendo el de mayor complejidad este último (Ramos \& Marín, 2018).
Para la identificación de problemas de intrusión de agua, existen diferentes métodos o particularmente registros que pueden hacer una detección temprana de invasión de agua y con esto un control prioritario, algunos de ellos son: registros de temperatura, medidores de tasa de flujo, registros gradiomanómetro, $\mathrm{BHC}, \mathrm{CBL}$ y los VDL.

La industria del petróleo en el fenómeno de producción elevada de agua controles de diferentes tipos como mecánicos y químicos o también selectivos y no selectivos. Dentro de los tratamientos selectivos se tienen bloqueadores de permeabilidad, reductores de permeabilidad, modificadores de permeabilidad relativa y no selectiva como gelificantes. Los anteriores controladores están basados en productos químicos como surfactantes, polímeros derivaros de resina de madera, metil, silicatos, etil, poliacrilamidas, entre otros. De acuerdo al problema que esté generando la elevada intrusión de agua, será el tratamiento de control a implementar.

Cuando el agua en exceso es obtenida en superficie puede dársele diferentes disposiciones o usos finales como vertimientos, inyección a pozos productores, disposal, uso para riego, uso industrial, reutilización en la industria, entre otros. De acuerdo al uso final que se determine se realizará un tratamiento que permita obtener las condiciones necesarias para dicho fin, puesto que se deben tener en cuenta los posibles contaminantes que tienen algunos de ellos son los sólidos presentes, aceites y grasas, compuestos orgánicos disueltos, metales pesados, productos químicos, entre otros.

La industria del petróleo ha buscado poder reutilizar las aguas producidas dentro de su misma operación, es así como la emplea en preparación de fluidos, reinyección para procesos de recuperación, inyección disposal para evitar depletamientos o hundimientos del subsuelo, recirculación entre otras fases propias del proceso de explotación de hidrocarburos.

\section{Referencias Bibliográficas}

1. Ahmed, T. (2010). Reservoir Engeneering Handbook . Oxford: Gulf Prodessional Publishing.

2. Arnold, Burnett, Elphick, Feeley, Galbrun \& Otros. (2004). Manejo de la producción de agua: De residuo a recurso. Schlumberger: Oilfield Review, 30-44

3. Baily, B., Crabtree, M., Tyrie, J., Elphick, J., Kuchuk, F., Romano, C., \& Roodhart, L. (2000). Control de Agua . Schulmerger: Oilfield Review, 32-53. 
4. Carrillo Pérez, J. (2010). Diseño Conceptual de una Planta Para el Tratamiento y Disposición Final de Aguas de Producción, Provenientes del Campo Aguasay en el Estado Monagas. Venezuela : Universidad de Oriente.

5. Carvalhinho Grimaldi, M., Castrisana, W., Coradini Tolfo, F., Protti Christino, F., Limoeiro Geraldo , L. M., Coutinho Saliba, G., \& Bastos Lopes, D. E. (2010). Produced Water Reuse for Production of Chemicals. Rio de Janeiro - Brazil : Society of Petroleum Engineers.

6. Chan, K.S. (1995). Water Control Diagnostic Plots. Paper SPE 30775. SPE Annual Technical Conference and Exhibition, Dallas.

7. Colorado Energy Office, \& University Water Center - (2014). Produced Water Beneficial Use Dialogue: Opportunities and Challenges for Re-Use of Produced Water on Colorado's Western Slope. Colorado.

8. Cortes Mejia, V. M. (2008). Control De La Producción De Agua En Pozos Petroleros . México, D.F.: Universidad Nacional Autonoma De México.

9. Datta-Gupta, A., Vasco, D.W., and Long, J.C.S. (1995) Sensitivity and Spacial Resolution of Transient Pressure and Tracer Data for Heterogeneity Characterization. Paper SPE 30589. SPE Annual Technical Conference and Exhibition, Dallas.

10. Ebratth Salgado, O. A. (18 de Enero de 2011). Análisis del Agua de Producción. Obtenido de Universidad Industrial de Santander : https:// es.slideshare.net/oebratth/agua-de-produccion.

11. Elphick, J. and Seright, R.S. (1997). A Classification of Water Problem Types. Paper 1. PNC 3rd International Conference on Reservoir Conformance, Profile Control, Water and Gas Shut Off, Houston.

12. ERCB, E. R. (2003). Energy Resources Conservation Board Directive 046: Production Audit Handbook. Canada: Alberta Energy Regulator.

13. Espinoza Okamoto , J. A. (2007). Tecnicas y Mejoras en las Mediciones de los Registros de Secrorización de Adherencia de Cemento (SBT). Lima- Peru: Universidad Nacional de Ingenieria.

14. F.T. Tao, Curtice, S., Hobbs , R., Sides, J., Wieser, J., Tuohey, D., \& Pliger, P. (1993). Conversion of Oilfield Produced Water Into an Irrigation/ Drinking Quality Water. San Antonio Texas: Society of Petroleum Engineers.

15. Faber. M.J. et al. (1998). Water Shut-Off Field
Experience with a Relative Permeability Modification System in the Marmul Field (Oman). Paper SPE 39633. Improved Oil Recovery Symposium, Tulsa.

16. Fulleylove, R.J. et al. (1996). Water Shut-Off in Oil Production Wells-Lessons from 12 Treatments. Paper SPE 36211. Petroleum Exhibition and Conference, Abu Dhabi.

17. Galvis Briñez, D. (2011). Operación de Campos Petroleros en Entornos Sostenibles: Un Enfoque en Aguas de Producción Petrolera. Bogotá: Ecopetrol.

18. Garcia, E. (s.f.). Caudal Limite Economico $y$ Productivo. Obtenido de https://es.scribd. com/document/56968525/Caudal-LimiteEconomico-y-Productivo.

19. Glover , P. (2008). Petrophysics. Reino Unido: University of Aberdeen.

20. Gomez Angeles, R. L. (2011). Tratamiento y Disposición Final de Aguas de Producción en el Nor-Este Peruano . Lima-Peru : Universidad Nacional de Ingeniería .

21. Guirguis, M. (2004). Treatment of Waste Water: A Reed Bed-Environmental Case History. Alberta - Canada : Society of Petroleum Engineers.

22. Halliburton Company, (1996). Conformance technology: identification and treatment of water-control problems for improved reservoir recovery efficiency, Houston:Estados Unidos, Halliburton Energy Services,

23. Hild, G.P. and Wackowski, R.K. (1999). Reservoir Polymer Gel Treatments to Improve Miscible CO2 Flood. SPEREE, 196-204.

24. Khamis, B., \& Haridas, B. (2003). High-water Cut: Experience And Assessment in ODO. Society of Petroleum Engineers, 1-7.

25. Khatib, Z., \& Verbeek, P. (2002, January 1). Water to Value-Produced Water Management for Sustainable Field Development of Mature and Green Fields. Society of Petroleum Engineers. doi: 10.2118/73853-MS

26. Krishnan, P. et al. (2000). Dehydration and Permeability of Gels Used in In-Situ Permeability Modification Treatments. Paper SPE 59347. SPE/DOE Improved Oil Recovery Symposium, Tulsa.

27. Kunchuk, F., Sengul, M., \& Zeybek, M. (1999). Oilfield Water: a Vital Resource. Middle East Well Evaluation Review, 5-13.

28. Lai, Q.J. et al. (1999). Gel-Cement Combination Squeezes for Gas Shutoff. Paper SPE 54596. SPE Western Regional Meeting, Anchorage. 
29. Lastockin, D., Wright, I., Allsopp, K., Dakers, M., \& Kantzas , A. (2004). Low Field NMR Water Cut Metering. Journal of Canadian Petroleum Technology, 17-21.

30. Lazarde, H. (2004). Interpretación de Perfiles de Producción. Venezuela : ESP OIL Engineering Consultants .

31. Macrae, J. (1997). Zero Extrusion Casing Patch. Paper SPE 37650. Drilling Conference, Amsterdam.

32. Marca, C. (1990). Remedial Cementing. Developments in Petroleum Science 28. Amsterdam.

33. Martinez, J., \& Konopczynski , M. (October de 2002). Integrated Reservoir Management in an Intelligent Well Environment. Society of Petroleum Engineers.

34. Meek, J.W. and Harris, K. L. (1991). Repairing Casing Leaks Using Small-Particle-Size Cement. Drilling Conference, Amsterdam.

35. Moreno Vázquez, B. M. (2011). Aplicación e Interpretación del Registro Sónico Compensado BHC. México: Instituto Politecnico Nacional Escuela Superior de Ingenieria y Arquitectura.

36. Mukerji, P. (2013). Production Logging Principles . Oildfield Review - Schlumberger.

37. Paris de Ferrer, M. (2001). Inyección de Agua y Gas en Yacimientos Petroliferos. Maracaibo: Venezuela: Ediciones Astro Data S.A.

38. Pérez, E. R., Santos Santos, N., \& Rojas, J. (2010). Determinación Teórica y Experimental de Permeabilidad Relaticas de Tres Fases. El Reventon Energético, 7-18.

39. Porras Tirado, J. A., \& Suarez Medina , J. F. (2016). Evaluación Técnico Financiera de un Sistema de Levantamiento Combinado Bombeo Mecánico e Hidráulico Para la Obtención de Información del Yacimiento por Medio de Registros de Producción en un Pozo en Campo Provincia . Bogotá: Fundación Universidad de América.

40. Rodríguez González, J. X., Cabrera Pilco , E. A., \& Condo Ramos, L. B. (2014). Factores Que Inciden en la Alta Producción de Agua en Campos Del Oriente Ecuatoriano y Posibles Soluciones. Guayaquil, Ecuador: Escuela Superior Politécnica del Litoral.

41. Root, P.J. and Skiba. F.F. (1965). Crossflow Effects during an Idealized Displacement Process in a Stratified Reservoir. SPEJ, 229-237.

42. Seldal, M. (1997). Using Water/Oil Ratios to Diagnose Excessive Water Production Mechanisms. New Mexico Institute of Mining and Technology.
43. Seright, R.S. (1988). Placement of Gels to Modify Injection Profiles. Paper SPE/DOE 17332. SPE/DOE Enhanced Oil Recovery Symposium, Tulsa.

44. Seright, R.S. (1993). Effect of Rock Permeability on Gel Performance in Fluid-Diversion Applications. In Situ, 363-386.

45. Seright, R.S. (2000). Gel Propagation through Fractures. Paper SPE 59316. SPE/DOE Improved Oil Recovery Symposium, Tulsa.

46. Seright, R.S., Liang, J., and Seldal, M. (1998). Sizing Gelant Treatments in Hydraulically Fractured Production Wells. SPEPF, 223-229.

47. Shewchuk, P.G. (1978). Alternatives of Wastewater Disposal to Direct Stream Discharge Including Land Utilization. Alberta Environment.

48. Smith, L.R., Fast, C.R., and Wagner, O.R. (1969). Development and Field Testing of Large Volume Remedial Treatments for Gross Water Channeling. JPT, 1015-1025.

49. Society of Petroleum Engieer. (2011). Challenges in Reusing Produced Water.

50. Society of Petroleum Engieer. (2011). Challenges in Reusing Produced Water.

51. Soliman, M.Y. et al. (2000). Integration of Technology Supports Preventive Conformance Reservoir Techniques. Paper SPE 62553. Western Regional Meeting, Long Beach.

52. Sondex Wireline Ltd. (2006). Standard Length And Short Compact Production Logging Toolstrings. 1-24.

53. Sorbie, K.S. and Seright, R.S. (1992). Gel Placement in Heterogeneous Systems with Crossflow. Paper SPE 24192. Symposium on Enhanced Oil Recovery, Tulsa.

54. Suarez, A., Jaramillo, M. d., Gonzaléz, A., Londoño, J. G., \& Pacavita, J. P. (2016). Gestión Integral de Recursos Hidricos. Bogotá: Ecopetrol.

55. Suarez, A., Gonzaléz, A., Londoño, J. G., \& Pacavita, J. P. (2017). Gestión Integral de Recursos Hidricos. Bogotá: Ecopetrol.

56. Sydansk, R.D. (1993). Acrylamide-Polymer/ Chromium (III) Carboxylate Gels for Near Wellbore Matrix Treatments. SPE Advanced Technology Series, 146-152.

57. Urdahl, H. et al. (1992). Experience with Temporarily Sealing Leaking Tubing Annuli with Extended-Life Polymer Gel Plugs in the Greater Ekofisk Area. Paper SPE 24982. European Petroleum Conference, Cannes, France. 
58. Veil JA, Puder M, Elcock D y Redweik R Jr. (2004). A White Paper Describing Produced Water from Production of Crude Oil, Natural Gas, and Coal Bed Methane.

59. Wagner, O.R. (1977). The Use of Tracers in Diagnosing Interwell Reservoir Heterogeneities-Field Results. JPT, 1410-1416.

Recepción: 27 de noviembre de 2018

Aceptación: 5 de Diciembre de 2019 